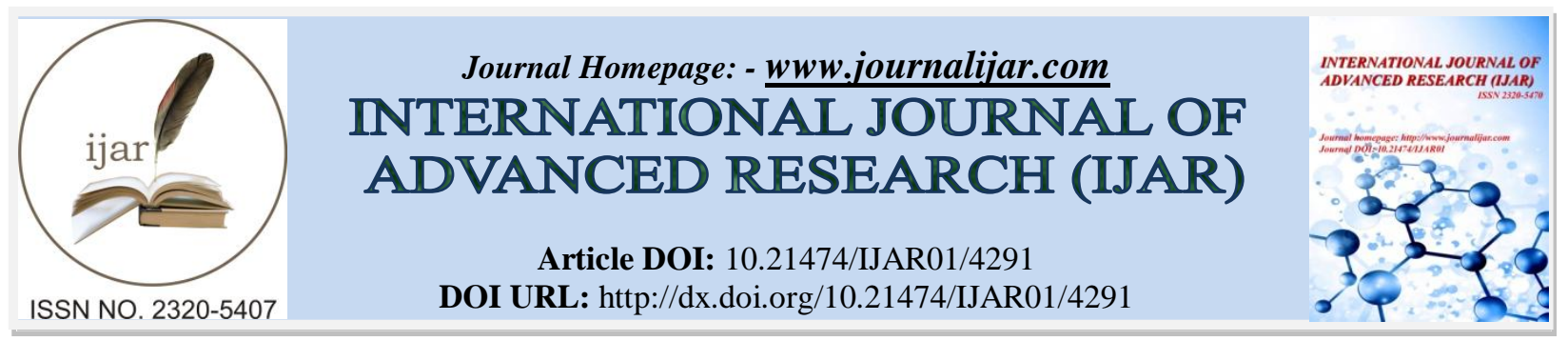

RESEARCH ARTICLE

\title{
EFFECTS OF STEEPING CONDITIONS (TEMPERATURE, PARTICLE SIZE AND DURATION) USING WATER ON STEVIOL GLYCOSIDES CONTENT FROM Stevia rebaudiana AND SENSORY EVALUATION OF OPTIMISED SAMPLE.
}

A. H. Nur Sabreena ${ }^{1}$, ${ }^{*}$. Nor Azma ${ }^{1}$, A. Asmah ${ }^{1}$ and O. Mohamad ${ }^{2}$.

1. Faculty of Plantation and Agrotechnology, Universiti Teknologi MARA, 40450 Shah Alam, Selangor, Malaysia.

2. Faculty of Agricultural, Universiti Putra Malaysia, 43400 Serdang, Selangor, Malaysia.

\section{Manuscript Info}

Manuscript History

Received: 28 March 2017

Final Accepted: 30 April 2017

Published: May 2017

\section{Abstract}

The influence of commonly used method which is steeping on temperature, size of leaves, as well as duration and hence the sensory evaluation of Stevia extract were studied. 20 of Stevia samples from China immersed in water as a medium of extraction by following the test variables suggested by RSM. The content of steviol glycosides in the crude extract was analysed with the utilization of refractometer and HPLC. The result shows that increasing of temperature and time of immersion, lead to higher content of Stevia compound meanwhile increasing of particle size lead to lower content of Stevia compound. Before conducted the sensory test on the optimised sample, ICP analysis was performed to check on the heavy metal content, the result obtained was $0.02 \mathrm{ppm}$ for mercury, $\mathrm{Hg}$ and $0.03 \mathrm{ppm}$ for lead, $\mathrm{Pb}$. In addition, $\mathrm{pH}$ for the extract was conducted and the result was 5.59. The sensory evaluation was conducted using 9-point hedonic scale towards 30 correspondents. A Mann Whitney test revealed that, there was insignificant of $p$-value for sweetness attributes with 0.4499, meanwhile for both bitterness and aftertaste gave significant $p$-values which were 0.0256 and 0.0041 respectively.

Copy Right, IJAR, 2017,. All rights reserved.

\section{Introduction:-}

Stevia is a small perennial shrub that has been used for centuries as a bio-sweetener and for other medicinal uses such as to lower blood sugar (Goyal \& Goyal, 2010). There are several chemical constituents that can be derived from the plant $S$. rebaudiana leaves such as diterpenoid ent-Kaurene, labdane, flavonoid, sterol, triterpenoid and other organic components. (De, Mondal, \& Banerjee, 2013). However, the best known are the diterpenoid glycosides which consist of stevioside, reb A, C, D, E, F and dulcoside A The sweet taste glycosides have been reported to be present in the leaves, flowers and stems but not in the roots of $S$. rebaudiana (De et al., 2013). There were three test variables have been chosen which were immersion temperature $\left({ }^{\circ} \mathrm{C}\right)$, particle size $(\mathrm{mm})$, and immersion duration (min). Those parameters suggested were among the most important factors that affect the extraction of Stevia have been explored in several studies (Abou-Arab, Abou-Arab, \& Abu-Salem, 2010; Afandi, Sarijan, \& Shaha, 2013). 
To reduce the usage of organic solvents, water is a feasible green solvent extraction. Hence, steeping method using water has become an efficient and low cost method of extraction for less-polar organic components from environmental soil, sediments and plant materials. Water is a highly polar solvent with a high dielectric constant $(\varepsilon)$ at room temperature and atmospheric pressure due to the presence of extensive hydrogen-bonded structure. Hence, traditionally water is not considered as a suitable extraction fluid for non-polar or organic compounds at room temperature. When the temperature of water is raised, there is a steady decrease in its permittivity, viscosity and surface tension but an increase in its diffusivity characteristics. Under these conditions, water behaves like certain organic solvents which can dissolve a wide range of medium and low polarity analytes (Teo, Tan, Yong, Hew, \& Ong, 2010).

The optimised samples was obtained and verified using RSM will be tested via sensory evaluation. The sample was evaluated using the performance of a hedonic scale for product acceptability. The most common scale for acceptance testing is the 9-point hedonic scale that was developed in the United States (US) by Peryam and Pilgrim in 1957 (Yeh et al., 1998). Hedonic scales have been used for a number of years. The 9-point hedonic scale is a balanced bipolar scale around neutral at the center with four positive and four negative categories on each side. The categories are labeled with phrases representing various degrees of affect and those labels are arranged successively to suggest a single continuum of likes and dislikes (Peryam \& Pilgrim, 1957).

\section{Materials and Method:- \\ Materials:-}

Dried leaves of Stevia bought from China through the Stevia Sugar Coorporation in Distilled water which has been filtered using filteration set, water bath from Lab Tech. Refractometer was purchased from Surechem Sdn Bhd (Sri Petaling, Malaysia).

\section{Methods:-}

\section{Extraction of Stevia:-}

The Stevia was extracted by following three parameters that has been chosed which were immersion temperature $\left({ }^{\circ} \mathrm{C}\right)$, Particle size $(\mathrm{mm})$, and Immersion duration $(\mathrm{min})$. An amount of $1 \mathrm{~g}$ of dried leaves was extracted with 100 $\mathrm{mL}$ distilled water using steeping method. Water bath and thermometer are used to control the temperature of extract. The crude extract was then filtered using Whatman filter paper No. 1.Distilled water act as a control in this experiment.

\section{Determination using Refractometer (\% Brix):-}

After the extraction process, the crude extract will be analyzed using refractometer to detect the total soluble solid. The range of refractometer that has been used was from $0 \%$ to $56 \%$. The refractometer need to be calibrated after reading first sample to avoid error in reading.

\section{ICP- Analysis:-}

$0.5000 \mathrm{~g}$ of Stevia leaves (China and MS012) were weighed and then put in a crucible silica. The samples were then baked in a muffle furnace at $550{ }^{\circ} \mathrm{C}$ for 6 to 7 hours and let them to be cooled overnight. After that, the samples were moved onto sandbath and $2 \mathrm{~mL}$ of $\mathrm{HCl}$ was added. Let them to be dried and $10 \mathrm{~mL}$ of $20 \% \mathrm{HNO}_{3}$ was added. After $1 / 3$ of the acid solution was dried, hence the remaining acid was filtered into a $50 \mathrm{~mL}$ volumetric flask.

\section{Sensory evaluation via Hedonic:-}

In order to analyze the optimised sample in the aspect of taste and human preference, human panel taste has to be conducted by using the hedonic scale rating. This is the most obvious choice, as it directly measures the effect one is trying to achieve, and consequently is the method most commonly used in product development. The panel testing is a psychophysical rating of gustatory stimuli. In this method, a group of about 20 to 30 human volunteers were required. The 9-point hedonic scale is a balanced bipolar scale around neutral at the center with four positive and four negative categories on each side.

\section{Results And Discussion:- \\ Extraction of Stevia:-}

The proposed methods, namely steeping showed that Stevia compound, could be extracted at elevated temperature using water without the addition of organic solvent. Table 1 shows the result of Stevia extraction using water which have been analysed via refractometer (\% Brix) and HPLC. It involved three variables which were immersion 
temperature, particle size and immersion duration. The 20 samples was optimised using RSM from the previous paper (A.H. Nur Sabreena, 2017).

Table 1:- Result of Stevia extraction.

\begin{tabular}{|c|c|c|c|c|c|}
\hline \multirow{2}{*}{ No } & \multicolumn{3}{|c|}{ Test variables } & \multicolumn{2}{c|}{ Responses } \\
\cline { 2 - 6 } & Temperature $\left({ }^{\circ} \mathbf{C}\right)$ & Particle size $(\mathbf{m m})$ & Duration $(\mathbf{m i n})$ & TSS (\%) & FITS \\
\hline 1 & 25 & 1 & 2 & $1.0 \pm 0.17$ & $1.08933 \pm 0.1$ \\
\hline 2 & 75 & 1 & 2 & $1.2 \pm 0.1$ & $1.40352 \pm 0.1$ \\
\hline 3 & 25 & 2 & 2 & $0.5 \pm 0.1$ & $0.42027 \pm 0.2$ \\
\hline 4 & 75 & 2 & 2 & $0.8 \pm 0.17$ & $0.91507 \pm 0.05$ \\
\hline 5 & 25 & 1 & 4 & $1.2 \pm 0.17$ & $1.34642 \pm 0.05$ \\
\hline 6 & 75 & 1 & 4 & $1.3 \pm 0.1$ & $1.47930 \pm 0.07$ \\
\hline 7 & 25 & 2 & 4 & $0.6 \pm 0.1$ & $0.65798 \pm 0.1$ \\
\hline 8 & 75 & 2 & 3 & $0.8 \pm 0.1$ & $0.97217 \pm 0.05$ \\
\hline 9 & 8.0 & 1.5 & 3 & $1.0 \pm 0.2$ & $0.95089 \pm 0.06$ \\
\hline 10 & 92 & 1.5 & 3 & $1.7 \pm 0.17$ & $1.56061 \pm 0.1$ \\
\hline 11 & 50 & 0.66 & 3 & $0.5 \pm 0.1$ & $1.40991 \pm 0.1$ \\
\hline 12 & 50 & 2.34 & 1.3 & $1.1 \pm 0.1$ & $0.980088 \pm 0.1$ \\
\hline 13 & 50 & 1.5 & 4.7 & $1.5 \pm 0.17$ & $1.24720 \pm 0.07$ \\
\hline 14 & 50 & 1.5 & 3 & $1.5 \pm 0.1$ & $1.51057 \pm 0.1$ \\
\hline 15 & 50 & 1.5 & 3 & $1.5 \pm 0.1$ & $1.51057 \pm 0.1$ \\
\hline 16 & 50 & 1.5 & 3 & $1.5 \pm 0.1$ & $1.51057 \pm 0.1$ \\
\hline 17 & 50 & 1.5 & 3 & $1.5 \pm 0.1$ & $1.51057 \pm 0.1$ \\
\hline 18 & 50 & 1.5 & 3 & $1.5 \pm 0.1$ & $1.51057 \pm 0.1$ \\
\hline 19 & 50 & 1.5 & 3 & $1.5 \pm 0.1$ & $1.51057 \pm 0.1$ \\
\hline 20 & 50 & 1.5 & & & \\
\hline
\end{tabular}

Previously, a group of researchers conducted an experiment of Stevia extraction using hot water. The variables involved were water ratio, time and temperature. The experiment also conducted an optimisation processes to achieved favourable condition in order to obtained high content of stevioside. The optimum conditions were leaf to water ratio was $1: 14(\mathrm{~g}: \mathrm{mL})$, temperature of water at $78^{\circ} \mathrm{C}$ and time of heating was 56 min (Rai, Majumdar, \& De, 2012).

Effects of Temperature:-

Figure 1 shows the effect of temperature on Stevia extraction. Elevation in temperature leads to increasing of the extraction yields of compounds. Although higher temperatures had positive effects on the extraction yields, temperature cannot be increased indefinitely, because of the instability of compounds. For example, partial hydrolysis of polysaccharides and denaturation of membranes may take place at certain temperatures (Cacace \& Mazza, 2003).

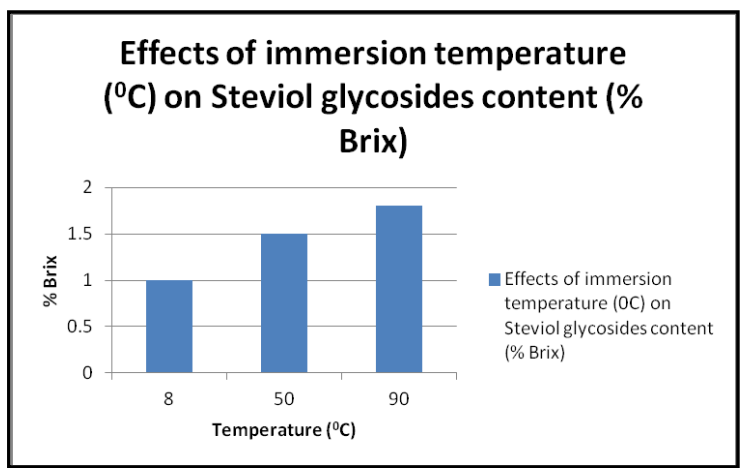

Figure 1:- Graph of the effect of immersion temperature on Stevia extraction. 
Temperature is the main factor which could affect the extraction efficiency. It could influence the physicochemical properties of water and also subject thermally labile analytes to their decomposition or hydrolytic attack (Smith, 2002). Besides, the high temperatures have also changed the properties of water and thus making the polarity of water closer to those of non-polar compounds. This will enhance the solubility of less polar compounds in water for extraction (Teo et al., 2010).

\section{Effects of particle sizes:-}

Figure 2 indicated the effect of particle size on Stevia extraction. Smaller size of the leaves lead to higher compound content of Stevia extract. The results of this study show that the particle size of the leaves influence the Stevia compositions. In 2003, a group of researchers conducted an experiment on the extraction of antioxidants from Balm (Melissa officinalis L.) leaves with ethanol. The study claimed that the total yield of extraction increases with the decrease of particle size. These results indicate that, in the latter stage of the extraction process, the diffusion of the solvent into the particle and the solvent-solute diffusion out of the particle are rate-governing steps of the process. (Herodež, Hadolin, Škerget, \& Knez, 2003).

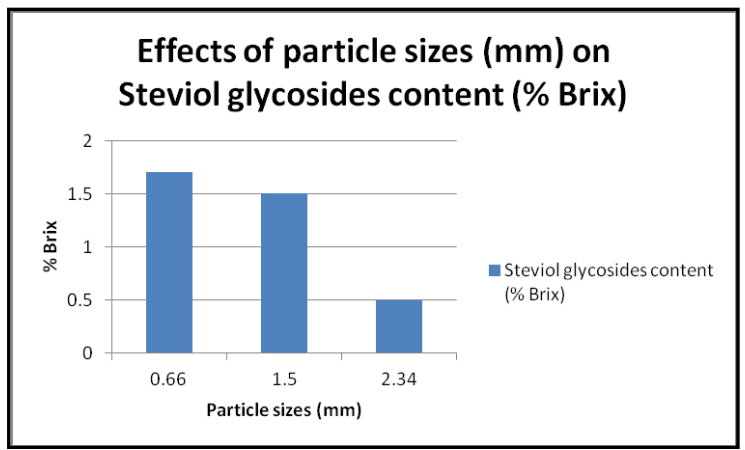

Figure 2:- Graph of the effect of particle size on Stevia extraction

\section{Effects of Duration:-}

Figure 3 pointed that the longer immersion duration takes place the higher content of Stevia extracts. These findings further support the idea of the previous study that, it was observed that a longer extraction time would result in a better yield for stevioside and reb A when extraction time increased (Teo, Tan, Yong, Hew, \& Ong, 2009).

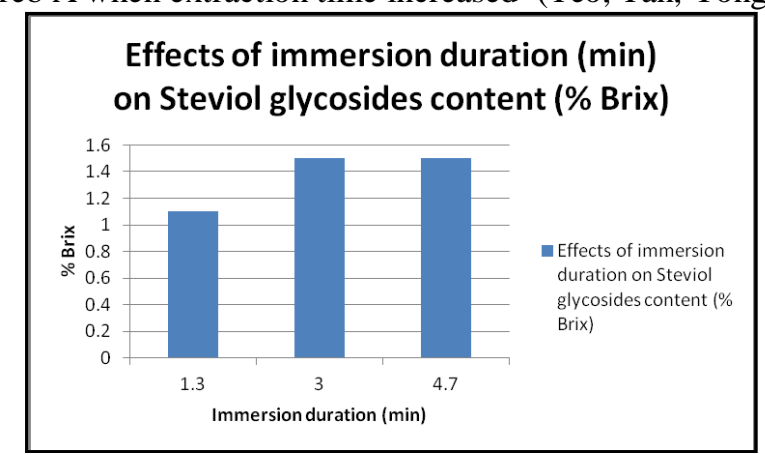

Figure 3:- Graph of the effect of immersion duration on Stevia extraction.

\section{Sensory Evaluation:-}

Before the sensory test was conducted, the ICP analysis and $\mathrm{pH}$ test was performed in order to ensure the safety of the extracts formed. ICP analysis was performed to check on the heavy metal content, the result obtained was 0.02 ppm for mercury, $\mathrm{Hg}$ and $0.03 \mathrm{ppm}$ for lead, $\mathrm{Pb}$. In addition, $\mathrm{pH}$ for the extract was conducted and the result was 5.59. Besides, the sensory test was conducted towards the optimised sample from previous research which was immersion temperature of $92^{\circ} \mathrm{C}$, particle size $0.7 \mathrm{~mm}$ and immersion duration of 4.7 minutes (A.H. Nur Sabreena, 2017). 
Table 2:- Result of Sensory evaluation

\begin{tabular}{|c|c|c|c|c|c|c|}
\hline \multirow{2}{*}{$\begin{array}{c}\text { Sample } \\
\text { Attributes }\end{array}$} & \multicolumn{3}{|c|}{ Stevia } & \multicolumn{3}{|c|}{ Cane Sugar } \\
\hline & $\mathbf{A}$ & B & $\mathbf{C}$ & $\mathbf{A}$ & B & $\mathrm{C}$ \\
\hline \multicolumn{7}{|l|}{ Scale } \\
\hline 9 & 10 & 5 & 1 & 9 & 30 & 30 \\
\hline 8 & 12 & 9 & 9 & 15 & 0 & 0 \\
\hline 7 & 3 & 7 & 6 & 6 & 0 & 0 \\
\hline 6 & 3 & 5 & 4 & 0 & 0 & 0 \\
\hline 5 & 1 & 2 & 3 & 0 & 0 & 0 \\
\hline 4 & 1 & 1 & 1 & 0 & 0 & 0 \\
\hline 3 & 0 & 1 & 3 & 0 & 0 & 0 \\
\hline 2 & 0 & 0 & 1 & 0 & 0 & 0 \\
\hline 1 & 0 & 0 & 2 & 0 & 0 & 0 \\
\hline
\end{tabular}

A: Sweetness B : Bitterness C: Aftertaste

Based on the Table 3, by loking at the median column, the median engagement were higher for Stevia compared to fine sugar.The median difference between Stevia and fine sugar for those three attributes sweetness, bitterness and aftertaste were $-0.000,1.00$ and 2.00 respectively with $95.8 \%$ confidence interval. The Mann Whitney test did not show any statistically significant differences between Stevia and fine sugar for sweetness attribute. This proved from the $p$-value obtained (Table 3) which is more than 0.05. This insignificant value implied that both sweeteners showing the sweetness attribute almost at the same level. Stevia act as a natural sweetener also well known as sugar leaf, candy leaf and sweet leaf due to its sweet taste that estimately 300 times sweeter than cane sugar (Balaswamy, Rao, Rao, Nagender, \& Satyanarayana, 2014).

Table 3:- Mann Whitney test for three Attributes.

\begin{tabular}{|c|c|c|c|c|}
\hline & & \multirow{2}{*}{ Median difference } & Adjusted $\boldsymbol{p}$-value \\
\hline & Stevia & Sugar & -0.000 & 0.4499 \\
\hline A & 1.000 & 0.000 & 1.00 & 0.0256 \\
\hline C & 2.00 & 0.00 & 2.00 & 0.0041 \\
\hline
\end{tabular}

A: Sweetness B: Bitterness C: Aftertaste

Meanwhile, a Mann Whitney revealed that, for bitterness and aftertaste, adjusted $p$-value showing $p<0.05$. The values were 0.0256 and 0.0041 respectively. The result obtained was due to the bitter taste of Stevia extracts. Previous research has investigated that glycosides were purely bitter principles which commonly found in plants of the Genitiaceae family and though they are chemically unrelated but possess the common property of an intensely bitter taste (Doughari, 2012). Many studies have attempted to improve the taste of Stevia. However, Stevia has an incredibly sweet taste, but in its raw form it has a very significant liquorice essence to it. There was a report which ratified that Stevia is the sweetest plant in the world because the leaves part contain diterpene glycoside that has a sweet taste but it is not metabolized and contain no calories (Kumar, Jha, \& Singh, 2007).

\section{Conclusion:-}

Based on the results obtained, it suggested that the Stevia extraction was affected by those three parameters which were immersion temperature, particle size and immersion duration. Besides, the sensory test indicated that the sweetness level between Stevia and cane sugar were almost equal due to insignificant of $p$-value $(p>0.05)$, whereas for bitterness and aftertaste there were significant $p$-value between the sweeteners that can be referred in Table $\mathbf{2}$. Therefore, Stevia extraction required an improvement of taste in order to be chosen as a daily sweetener. However people that are concern about their health would chose Stevia for their drinks and foods as Stevia has a medicinal properties. 


\section{References:-}

1. A.H. Nur Sabreena, Y. N. A., O. Mohamad (2017). RESPONSE SURFACE METHODOLOGY FOR OPTIMISATION OF PARAMETERS FOR EXTRACTION OF Stevia rebaudiana USING WATER, H2O. IIOABJ, 8, 8.

2. Abou-Arab, A. E., Abou-Arab, A. A., \& Abu-Salem, M. F. (2010). Physico-chemical assessment of natural sweeteners steviosides produced from Stevia rebaudiana Bertoni plant. African Journal of Food Science, 4(5), 269-281.

3. Afandi, A., Sarijan, S., \& Shaha, R. K. (2013). Optimization of rebaudioside a extraction from Stevia Rebaudiana (Bertoni) and quantification by high perfomance liquid chromatography analysis. Journal of Tropical Resources and Sustainable Sciences, 1(1), 62-70.

4. Cacace, J., \& Mazza, G. (2003). Mass transfer process during extraction of phenolic compounds from milled berries. Journal of Food Engineering, 59(4), 379-389.

5. De, S., Mondal, S., \& Banerjee, S. (2013). Introduction to stevioside. Stevioside: Technology, Applications and Health, 1-25.

6. Doughari, J. H. (2012). Phytochemicals: Extraction methods, basic structures and mode of action as potential chemotherapeutic agents: INTECH Open Access Publisher.

7. Goyal, S., \& Goyal, R. (2010). Stevia (Stevia rebaudiana) a bio-sweetener: a review. International Journal of Food Sciences and Nutrition, 61(1).

8. Herodež, Š. S., Hadolin, M., Škerget, M., \& Knez, Ž. (2003). Solvent extraction study of antioxidants from Balm (Melissa officinalis L.) leaves. Food Chemistry, 80(2), 275-282.

9. Kumar, S., Jha, Y., \& Singh, P. (2007). Stevia: A natural potential source of sugar replacer. Beverage and Food World, 34, 70-71.

10. Peryam, D. R., \& Pilgrim, F. J. (1957). Hedonic scale method of measuring food preferences. Food technology.

11. Rai, C., Majumdar, G., \& De, S. (2012). Optimization of process parameters for water extraction of stevioside using response surface methodology. Separation Science and Technology, 47(7), 1014-1022.

12. Smith, R. M. (2002). Extractions with superheated water. Journal of chromatography A, 975(1), 31-46.

13. Teo, C. C., Tan, S. N., Yong, J. W. H., Hew, C. S., \& Ong, E. S. (2009). Validation of green-solvent extraction combined with chromatographic chemical fingerprint to evaluate quality of Stevia rebaudiana Bertoni. Journal of separation science, 32(4), 613-622.

14. Teo, C. C., Tan, S. N., Yong, J. W. H., Hew, C. S., \& Ong, E. S. (2010). Pressurized hot water extraction (PHWE). Journal of chromatography A, 1217(16), 2484-2494.

15. Yeh, L., Kim, K., Chompreeda, P., Rimkeeree, H., Yau, N., \& Lundahl, D. (1998). Comparison in use of the 9point hedonic scale between Americans, Chinese, Koreans, and Thai. Food Quality and Preference, 9(6), 413419. 